Research Article

\title{
Pull-In Analysis of the Flat Circular CMUT Cell Featuring Sealed Cavity
}

\author{
Wen Zhang, Hui Zhang, Fei Du, Jianchao Shi, Shijiu Jin, and Zhoumo Zeng \\ State Key Laboratory of Precision Measurement Technology and Instrument, Tianjin University, Tianjin 300072, China \\ Correspondence should be addressed to Hui Zhang; hzhang@tju.edu.cn
}

Received 27 January 2015; Revised 16 August 2015; Accepted 19 August 2015

Academic Editor: Filippo Ubertini

Copyright ( 2015 Wen Zhang et al. This is an open access article distributed under the Creative Commons Attribution License, which permits unrestricted use, distribution, and reproduction in any medium, provided the original work is properly cited.

\begin{abstract}
Capacitive micromachined ultrasonic transducers (CMUTs) are one of the appealing MEMS devices. Most studies treat CMUTs as rigid plates vibrating in open air, ignoring the mechanical boundary conditions for simplification and resulting in cumulative errors in coupled fields. This paper presents a new analytical model for the pull-in characteristics of the flat circular CMUT cell featuring sealed cavity. Utilizing the plate theory coupled with Boyle's law, the paper establishes a strong relation between the pressures inside the sealed cavity and the pull-in characteristics for the first time. Not only did we point out that the existence of the pressure inside the sealed cavity cannot be omitted, but we also quantified the direct effect of the pressure ratios on the pull-in phenomenon. The pull-in voltages increase while the pull-in ratios decrease with the pressure ratios of the pressure inside the sealed cavity to the ambient pressure. The proposed calculation process delivers a good approximation of the pull-in voltages and displacements, which are consistent with COMSOL simulation results. Particularly, the percentage error of our calculation process is $6.986 \%$ for the worst case. Therefore, our proposed analytical model accurately and efficiently predicts the pull-in characteristics and this paper offers new perspectives and reference value in designing and modeling the CMUTs.
\end{abstract}

\section{Introduction}

Research on MEMS (microelectromechanical systems) has seen an amazing growth during the last three decades, stimulated both by their interesting physical properties and their attractive application potential. One of those appealing MEMS devices is capacitive micromachined ultrasonic transducers (CMUTs) [1, 2]. Advantages of CMUTs over conventional piezoelectric transducers include better impedance matching with fluids, broader bandwidth, stable device properties, the ease of fabrication, and the possibility of on-chip integration with electronics [3]. Thus CMUTs are turning out to be a promising alternative in the fields of medical ultrasonic imaging, flow measurement, and aircoupled nondestructive applications [4].

Basically, the CMUT converts electrical energy into mechanical energy and vice versa [5]. Throughout the electromechanical coupling process, the pull-in characteristics are of great significance [6]. Different analyses and modeling approaches can be found in the literatures $[7,8]$. Most studies $[5,9,10]$ treat CMUTs as rigid plates in open air in their analysis, with a uniform velocity profile across the CMUT surface. Combining the electrostatic force and the elastic force, the approximate pull-in voltage could be obtained. This assumption, first issued by [5], was adopted because of its ease in modeling and the similarity to the theory used for piezoelectric transducers. However, considering the relative small dimensions of CMUTs, the cumulative effect of errors in coupled fields may lead to a huge deviation, and the reasons are as follows.

Firstly, the model does not take the top electrode into consideration for the static calculation, and the flexural rigidity depends on the dimensions and properties of the membrane only. Unlike the single-layer plate, the flexural rigidity of the moving parts depends on both the top electrode and the membrane, resulting in a homogenized flexural rigidity. Secondly, the model does not take into account the mechanical boundary conditions of the fixed circumference and simplifies the moving parts to be a uniform plate with exactly the same deflection and velocity throughout. Thirdly, the model neglects the significant effects of the pressure inside the sealed cavity. Once the cavity is hermetically sealed 
during the fabrication, the pressure inside is set. Under the external pressure and the biased voltage, the thin film inside the cavity will be squeezed and have an impact on the pull-in characteristics.

Our aim was to build up a calculation process of pullin characteristics considering the effects of the top electrode, the fixed boundary conditions, and the pressure inside the sealed cavity, which would be accurate enough for designing while avoiding a massive electromechanical Finite Element Modeling (FEM). Combining the plate theory featuring fixed circumference and Boyle's law inside the sealed cavity, this paper, for the first time, obtained a relation of the pullin characteristics with respect to the pressure ratios of the pressures inside the cavity to the ambient pressure.

Section 1 introduces the device and issues the main problems with former research. Details of the development of pull-in analysis are provided. Section 2 takes the top electrode into consideration and treats the CMUT as a flexible thin plate. Based on the fixed boundary conditions and Boyle's law, the mathematical descriptions of pull-in characteristics are obtained. In Section 3, the theoretical results provided by this model are compared with FEM results using COMSOL Multiphysics. The discussion involves an investigation of the key features of pull-in characteristics, including both pull-in ratios and pull-in voltages. Section 4 concludes the paper and assesses the regime of validity and limitations of the present model.

\section{Problem Formulation and Solution}

The typical CMUT cell is built with a circular, square, or hexagonal membrane separated from a fixed substrate by a small air gap [11]. The circular flat CMUT cell is chosen for its prevalence. Several thousand such cells electrically connected in parallel to form a CMUT array. The geometry of the CMUT cell is shown as Figure 1. The moving part of the CMUT cell is made from a silicon nitride membrane covered with a gold electrode [12]. A layer of silicon dioxide is deposited in order to prevent the electrical shortcut between the two electrodes. The highly doped silicon substrate is also utilized as the bottom electrode. Using wafer-bonding technology $[13,14]$, the cavity and the membrane are defined on separate wafers and then are bonded under vacuum conditions.

CMUTs are efficient transmitters and receivers for aircoupled nondestructive evaluation applications, generating or detecting ultrasonic waves by vibrating membrane featuring fixed circumference, respectively [15]. When a DC voltage is applied between the two electrodes, the membrane is attracted to the substrate by electrostatic forces. When an AC voltage is superimposed over the DC voltage, the membrane will move in response to the applied signal and an ultrasonic wave is generated and launched into the ambient fluid. Similarly, when an ultrasonic wave approaches the membrane kept under a DC bias, it will vibrate and an output $\mathrm{AC}$ voltage can be measured from the CMUT electrodes. The applied DC voltage is necessary because it supplies the charge that is modulated by the membrane movement to create an AC signal.

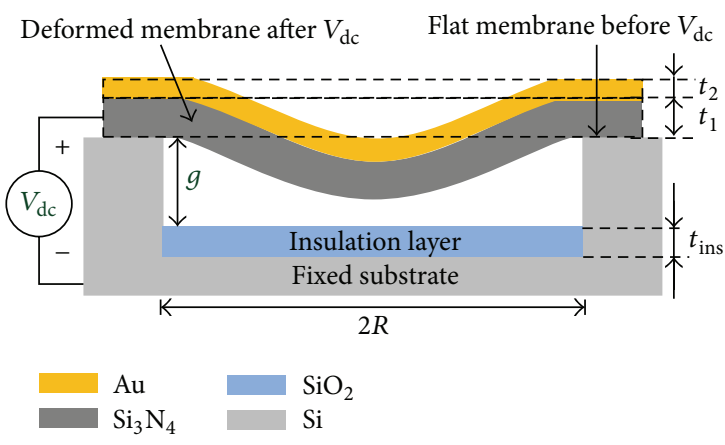

Figure 1: Geometry of a flat circular CMUT cell.

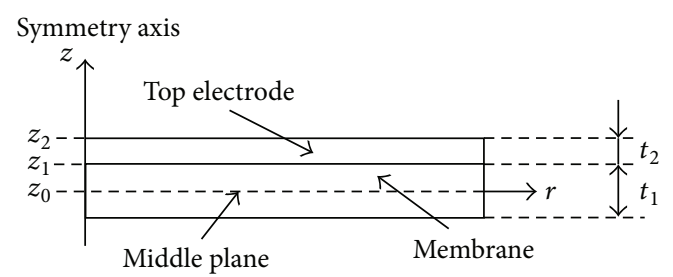

FIGURE 2: Schematic cross section of the flexural rigidity calculation.

2.1. Mechanical Modeling of a CMUT Cell. Taking the electrode into consideration, the flexural rigidity depends on the properties and dimensions of both the membrane and the electrode $[16,17]$. Thus, the flexural rigidity $D$ is given by

$$
D=\frac{1}{3} \sum_{i=1}^{2} \frac{E_{i}\left(z_{i}^{3}-z_{i-1}^{3}\right)}{1-v_{i}^{2}}
$$

where $E_{1}$ and $E_{2}$ are Young's Modulus of the membrane and the top electrode, respectively. $t_{1}$ and $t_{2}$ are their thicknesses. $v_{1}$ and $v_{2}$ are their Poisson's ratios. $z_{0}$ is the location of the median plane and $z_{i}$ is the location of the upper surface of the $i$ th layer, as shown in Figure 2.

Together with the top electrode and the membrane, the moving part of the CMUT cell could be taken as a flexible thin plate with radius $R$. The equation governing deflection of a thin flexible circular plate under a uniform loading $p$ is given as

$$
\frac{d}{d r}\left[\frac{1}{r} \frac{d}{d r}\left(r \frac{d w(r)}{d r}\right)\right]=\frac{p \cdot \pi r^{2}}{2 \pi r \cdot D}=\frac{p r}{2 D}
$$

Integrating several times [18] leads to

$$
\begin{aligned}
\frac{d w(r)}{d r} & =\frac{p r^{3}}{16 D}+\frac{C_{1} r}{2}+\frac{C_{2}}{r} \\
w(r) & =\frac{p r^{4}}{64 D}+\frac{C_{1} r^{2}}{4}+C_{2} \ln r+C_{3}
\end{aligned}
$$

where $C_{j}(j=1,2,3)$ are constants that can be evaluated from the boundary conditions. 
The CMUT cell is assumed to be clamped on the circumference; thus the boundary conditions are

$$
\begin{gathered}
\left.\frac{d w(r)}{d r}\right|_{r=0}=0, \\
\left.w(r)\right|_{r=R}=0, \\
\left.\frac{d w(r)}{d r}\right|_{r=R}=0 .
\end{gathered}
$$

Taking the boundary conditions (4) into (3) leads to

$$
\begin{aligned}
& C_{1}=-\frac{p R^{2}}{8 D}, \\
& C_{2}=0, \\
& C_{3}=\frac{p R^{4}}{64 D} .
\end{aligned}
$$

The vertical deflection $w(r)$ is obtained as

$$
w(r)=\frac{p R^{4}}{64 D}\left[1-\left(\frac{r}{R}\right)^{2}\right]^{2}=w_{\max }\left[1-\left(\frac{r}{R}\right)^{2}\right]^{2}
$$

and $w(r=0)=w_{\max }=p R^{4} / 64 D$.

Over the entire plate area, the average deflection could be defined as

$$
w_{\mathrm{avg}}=\frac{\int_{0}^{R} 2 \pi r \cdot w(r) d r}{\pi R^{2}}=\frac{p R^{4}}{192 D}=\frac{1}{3} w_{\max } .
$$

2.2. Electrical Modeling of a CMUT Cell. As shown in Figure 1, the membrane and the insulation layer make up a capacitor in series with the gap capacitance. Therefore, the effective gap distance is expressed as

$$
h=g+\frac{t_{\text {ins }}}{\varepsilon_{\text {ins }}},
$$

where $t_{\text {ins }}$ is the thickness of the insulation layer and $\varepsilon_{\text {ins }}$ the relative permittivity of the silicon dioxide.

When a DC voltage is applied between the electrodes, as shown in Figure 3, the electrical capacitance inside the sealed cavity could be calculated as

$$
\begin{aligned}
C & =\int_{0}^{R} \frac{2 \pi r \varepsilon_{0}}{h-w(r)} d r=\int_{0}^{R} \frac{2 \pi r \varepsilon_{0}}{h-w_{\max }\left[1-(r / R)^{2}\right]^{2}} d r \\
& =\frac{\varepsilon_{0} \pi R^{2}}{h} \int_{0}^{R} \frac{1}{1-\left(w_{\max } / h\right)\left[1-(r / R)^{2}\right]^{2}} d\left(\frac{r^{2}}{R^{2}}\right) \\
& =\frac{\varepsilon_{0} \pi R^{2}}{h} \cdot \frac{\operatorname{arctanh}\left(\sqrt{w_{\max } / h}\right)}{\sqrt{w_{\max } / h}},
\end{aligned}
$$

where $\varepsilon_{0}$ is the permittivity in the vacuum gap and $\varepsilon_{0}=8.85 \times$ $10^{-12} \mathrm{~F} / \mathrm{m}$. For simplicity, we denote $x=w_{\max } / h$. Then (9) could be rewritten as

$$
C=\frac{\varepsilon_{0} \pi R^{2}}{h} \cdot \frac{\operatorname{arctanh}(\sqrt{x})}{\sqrt{x}} .
$$

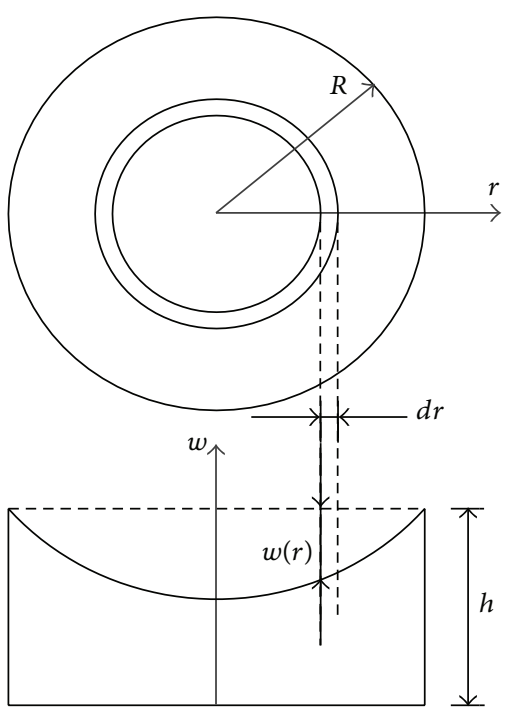

FIgURE 3: Illustration of the capacitance calculation.

The first derivative of $C$ with respect to $x$ is given by

$$
\frac{d C}{d x}=\frac{\varepsilon_{0} \pi R^{2}}{2 h} \cdot \frac{1}{x}\left(\frac{1}{1-x}-\frac{\operatorname{arctanh}(\sqrt{x})}{\sqrt{x}}\right) .
$$

The electrical force on the membrane caused by an applied voltage $V$ is given as [19]

$$
F_{e}=\frac{1}{2} V^{2} \frac{d C}{d w_{\mathrm{avg}}}=\frac{1}{2} V^{2} \frac{d C / d x}{d w_{\mathrm{avg}} / d x}=\frac{3 V^{2}}{2 h} \cdot \frac{d C}{d x} .
$$

2.3. Calculation of Pull-In Characteristics. Usually, the cavity between the membrane and the substrate is hermetically sealed during the fabrication, and the pressure inside the cavity has great effect on the deviation of the pull-in characteristics [13].

Suppose the ambient pressure is $p_{a}$. The initial pressure inside the cavity is $p_{c}=\alpha p_{a}$ and $0 \leq \alpha \leq 1$. Before the DC voltage is applied between two electrodes, the moving part has an initial deflection as shown in Figure 4(a). The equilibrium equation is

$$
F_{m 0}=k w_{\mathrm{avg} 0}=\left(p_{a}-p_{c}\right) \pi R^{2} .
$$

After the DC voltage is applied, the equilibrium equation changes to

$$
F_{m}=k w_{\mathrm{avg}}=\left(p_{a}-p_{c}^{\prime}\right) \pi R^{2}+F_{e},
$$

where $p_{c}^{\prime}$ is the pressure inside the cavity after the DC voltage, as shown in Figure 4(b).

Based on the analysis above, the process before and after the biased voltage should be isothermal yet not adiabatic. According to Boyle's law, the product of the pressure inside the cavity and the volume of the cavity are a constant; that is,

$$
p_{c} \pi R^{2}\left(g-w_{\mathrm{avg} 0}\right)=p_{c}^{\prime} \pi R^{2}\left(g-w_{\mathrm{avg}}\right) .
$$




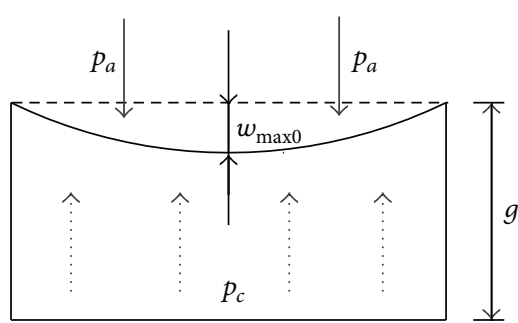

(a) $V=0 \quad w_{\text {avg } 0}=(1 / 3) w_{\max 0}$

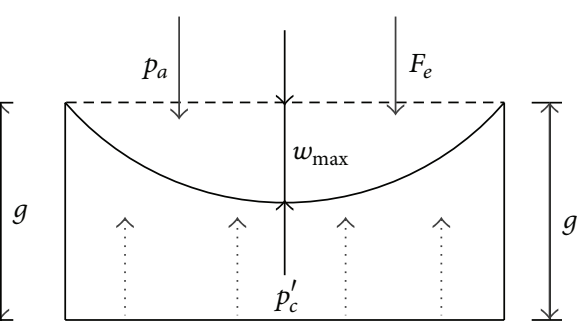

(b) $V \neq 0 \quad w_{\text {avg }}=(1 / 3) w_{\max }$

FIGURE 4: Illustrations before (a) and after (b) the DC voltage is applied.

Combining (7) and (13), the spring coefficient $k$ for the circular plate with fixed circumference could be obtained as

$$
k=\frac{192 \pi D}{R^{2}} .
$$

When a biased voltage is applied between the CMUT's electrodes, regardless of its polarity, it deflects the thin plate towards the bottom electrode. If the applied voltage is approaching the pull-in voltage, the plate will reach a stable state eventually. After several steps of calculation and iteration, the relation among the biased voltage $V$, the ratio $x$ of the central deflection to the effective gap distance, and the ratio $\alpha$ of the pressure inside the cavity to the ambient pressure could be obtained as

$$
V=\sqrt{\frac{4 h^{2} x\left[\left(64 \pi D h / R^{2}\right) x-\left(1-\left(\left(1-w_{\max 0} / 3 g\right) /(1-(h / 3 g) x)\right) \alpha\right) p_{a} \pi R^{2}\right]}{3 \varepsilon_{0} \pi R^{2}(1 /(1-x)-\operatorname{arctanh}(\sqrt{x}) / \sqrt{x})}} .
$$

This expression points out the relations among $V, x$, and $\alpha$. Under every pressure ratio $\alpha$, the equation has two digital roots. As the voltage increases, two solutions approach each other and coalesce together, as in Figure 5. This is the pullin point where the pull-in phenomenon occurs and the pullin characteristics could be obtained. In Figure 5, since the pressure inside the cavity is not zero $(\alpha=0.2)$, there will be initial vertical deflections; thus the ratio $x=w_{\max } / h$ does not start from 0 .

This paper focuses on the pull-in analysis of the flat circular CMUT cell featuring sealed cavity. In Section 3, in order to verify the new model for CMUTs without biased voltage, (6) and (7) will be used to calculate the maximum and average deflections of the CMUT's moving part as a flexible thin plate. Equation (17) will be used to calculate the pull-in characteristics under different pressure ratios for the validation of the proposed analytical model.

\section{Validation and Discussion}

For the validation of the present analytical model for the pullin characteristics, this section is organized as follows.

Firstly, in order to verify the accuracy of the new model in mechanical field only, the maximum deflections and the average deflections without biased voltage have been analyzed and modeled by the analytical model and FEM, respectively. For every pressure ratio $\alpha, w_{\max }$ and $w_{\text {avg }}$ obtained from the new model are compared with the FEM results.

Secondly, for the validation and accuracy of the new model in electromechanical coupled fields, the pull-in characteristics for different pressures inside the sealed cavity are calculated and modeled by the analytical model and FEM, respectively. For every pressure ratio $\alpha$, the pull-in voltage and the pull-in ratio predicted by the new model are compared with the FEM simulations. Besides, the pull-in characteristics obtained from the analytical model in [5] are also used for comparison.

The dimensions of the CMUT cell used in this section are listed in Table 1. The material properties needed for calculations and simulations are listed in Table 2.

Featuring small dimensions, the MEMS devices often involve complex interactions in coupled domains, and FEM is commonly used to deal with such nonlinear models. In this section, FEM simulations are taken as reference, with whom the analytical results are compared for the model's validation and accuracy.

We constructed a Finite Element Model of a CMUT cell in COMSOL Multiphysics (COMSOL Inc., Burlington, MA, USA) software, coupling the structural mechanics subdomain and the electrostatics subdomain. The $2 \mathrm{D}$ axisymmetric electromechanical coupling model is used, as shown in Figure 6. The free triangular mesh is used and the minimum mesh size is $0.00625 \mu \mathrm{m}$ as illustrated in Figure 7. The average mesh quality factor is 0.9274 , which is high enough for accurate simulations. As the bottom electrode, the fixed substrate of CMUT does not create any displacements, so it could be omitted when modeling. Instead, the interface between the bottom electrode and the insulation layer could be set for zero voltage for the electrostatics subdomain. The CPU of the workstation for FEM simulations in this paper is $\operatorname{Inter}(\mathrm{R})$ Xeon(R) CPU E5-2680 V3 @2.5 GHz, and the memory is 192 GB. 


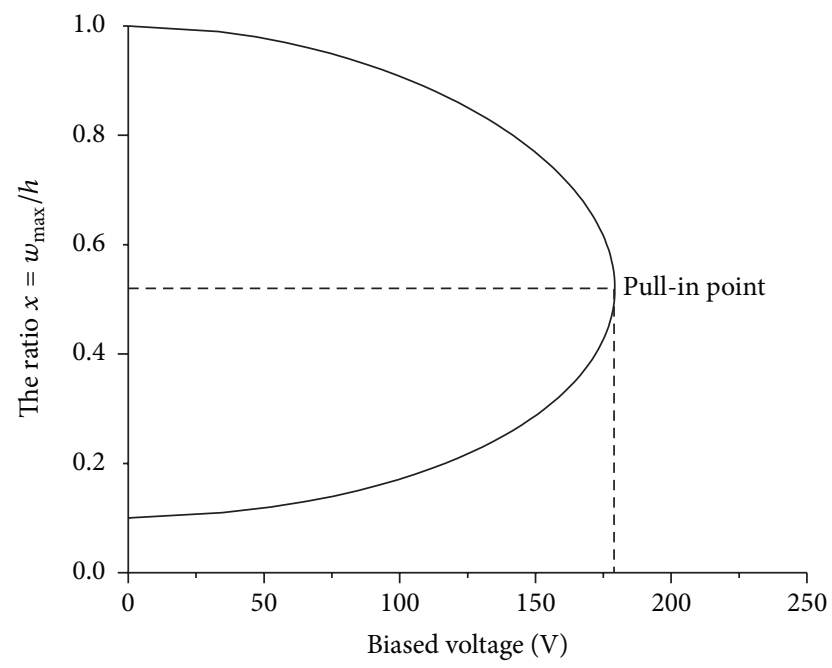

FIGURE 5: The voltage is plotted with respect to the ratio $x$ under $\alpha=0.2$.

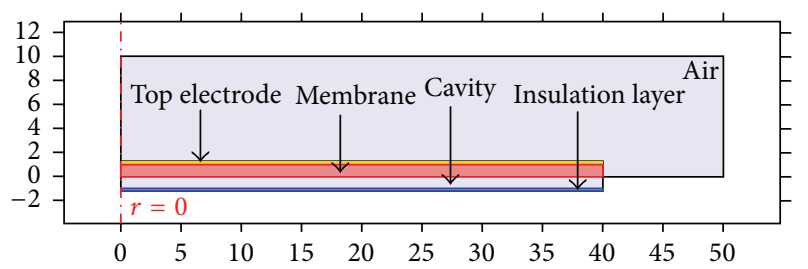

Figure 6: 2D axisymmetric FEM model of a CMUT cell.

TABLE 1: Dimensions of the CMUT cell $(\mu \mathrm{m})$.

\begin{tabular}{lcccc}
\hline$R$ & $t_{1}$ & $t_{2}$ & $t_{\text {ins }}$ & $g$ \\
\hline 40 & 1 & 0.3 & 0.15 & 1 \\
\hline
\end{tabular}

TABLE 2: Material properties of the CMUT.

\begin{tabular}{lccc}
\hline & $\mathrm{Au}$ & $\mathrm{Si}_{3} \mathrm{~N}_{4}$ & $\mathrm{SiO}_{2}$ \\
\hline Young's Modulus/GPa & 70 & 320 & 70 \\
Density/g/cm & 32 & 3.27 & 2.2 \\
Poisson's ratio & 19.3 & 0.263 & 0.17 \\
Relative permittivity & 0.44 & 5.7 & 4.2 \\
\hline
\end{tabular}

3.1. Displacement Comparison without Biased Voltage. Featuring the aforementioned dimensions, the CMUT cell is simulated under different pressure inside the sealed cavity. Without biased voltage, still, the membrane together with the top electrode would deflect due to the difference between the ambient pressure and the pressure inside the sealed cavity, as shown in Figure 8.

The maximum deflections and the average deflections for different pressures inside the sealed cavity are calculated by (6), (7) and simulated by FEM. The deflections and the relative errors between the analytical and FEM simulations are demonstrated in Figures 9 and 10. The black line stands for the data given by the new model, the red line stands for the FEM results, and the blue line represents the relative errors

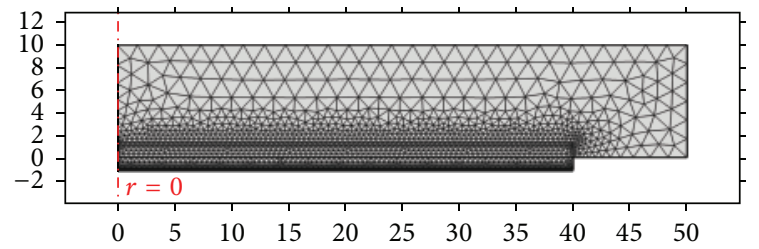

Figure 7: Mesh information of the 2D axisymmetric FEM model.

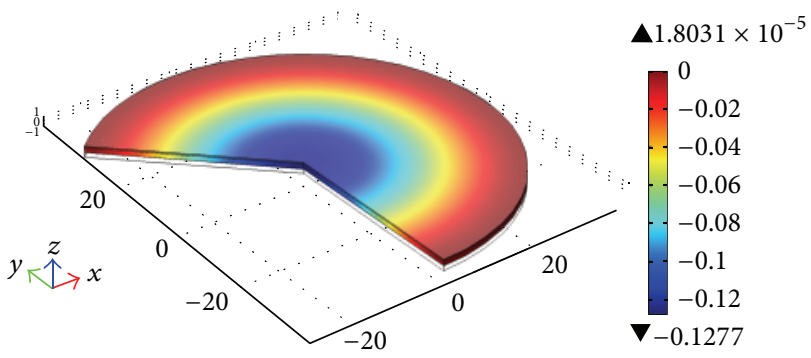

FigURE 8: Vertical displacement without biased voltage $(\alpha=0)$.

between the new model and the FEM. Relevant data are listed in Table 3.

From Figures 9 and 10, it can be obtained that the maximum deflections and the average deflections decrease as the pressure ratio $\alpha$ increases. Since $\alpha$ is the ratio of the pressure inside the sealed cavity to the ambient pressure $\left(\alpha=p_{c} / p_{a}\right)$, it also represents the pressure difference across the moving part. When the pressure inside the sealed cavity is smaller ( $\alpha$ is smaller), the deflections are bigger. Therefore, the moving part of the CMUT cell has bigger initial deflections with a smaller $\alpha$.

Together with Table 3, it can be observed that, for the mechanical field only, the new model could accurately predict the vertical deflections of the CMUT's moving part without biased voltage. The analytical predictions are in great agreement with the FEM results, yet the analytical deflections are slightly bigger. Also, the FEM results are taken as reference. The relative errors between the new model and FEM results for the maximum deflections are $0.850 \% \sim 2.964 \%$, and the relative errors for the average deflections are $0.006 \% \sim 2.073 \%$.

\subsection{Pull-In Comparison under Different Pressure Ratios}

3.2.1. Pull-In Ratios Comparison. To perform the parametric analysis in coupled fields, a range of pressure ratios are applied and the model is run through a sequence of static analyses for different parameter values. Every sequence follows through a certain number of iterations and the convergence is inspected. The process is repeated and the voltage is incremented until the membrane is about to contact the insulation layer. At this point, the solution ceases to converge and the simulation terminates. The last voltage value before divergence of the solution is the pull-in voltage. When the pull-in voltage is applied to the electrodes, we call the $w_{\max }$ then to be the pull-in gap and the $x=w_{\max } / h$ then to be 
TABLE 3: Comparisons of the maximum and average deflections without biased voltage.

\begin{tabular}{|c|c|c|c|c|c|c|}
\hline \multirow{2}{*}{$\alpha$} & \multicolumn{3}{|c|}{$w_{\max } / \mathrm{nm}$} & \multicolumn{3}{|c|}{$w_{\text {avg }} / \mathrm{nm}$} \\
\hline & New model & FEM & Relative error & New model & FEM & Relative error \\
\hline 0 & 131.475 & 127.690 & $2.964 \%$ & 43.825 & 42.935 & $2.073 \%$ \\
\hline 0.1 & 117.327 & 115.020 & $2.006 \%$ & 39.109 & 38.668 & $1.141 \%$ \\
\hline 0.2 & 103.180 & 102.310 & $0.850 \%$ & 34.393 & 34.391 & $0.006 \%$ \\
\hline 0.3 & 91.032 & 89.568 & $1.635 \%$ & 30.344 & 30.106 & $0.790 \%$ \\
\hline 0.4 & 78.885 & 76.805 & $2.708 \%$ & 26.295 & 25.814 & $1.862 \%$ \\
\hline 0.5 & 64.737 & 64.023 & $1.116 \%$ & 21.579 & 21.517 & $0.289 \%$ \\
\hline 0.6 & 52.590 & 51.226 & $2.662 \%$ & 17.530 & 17.215 & $1.827 \%$ \\
\hline 0.7 & 39.442 & 38.420 & $2.661 \%$ & 13.147 & 12.911 & $1.829 \%$ \\
\hline 0.8 & 26.295 & 25.609 & $2.678 \%$ & 8.765 & 8.606 & $1.846 \%$ \\
\hline 0.9 & 13.147 & 12.800 & $2.715 \%$ & 4.382 & 4.301 & $1.891 \%$ \\
\hline 1 & 0 & $4.2421 e-4$ & - & 0 & 0.002 & - \\
\hline
\end{tabular}

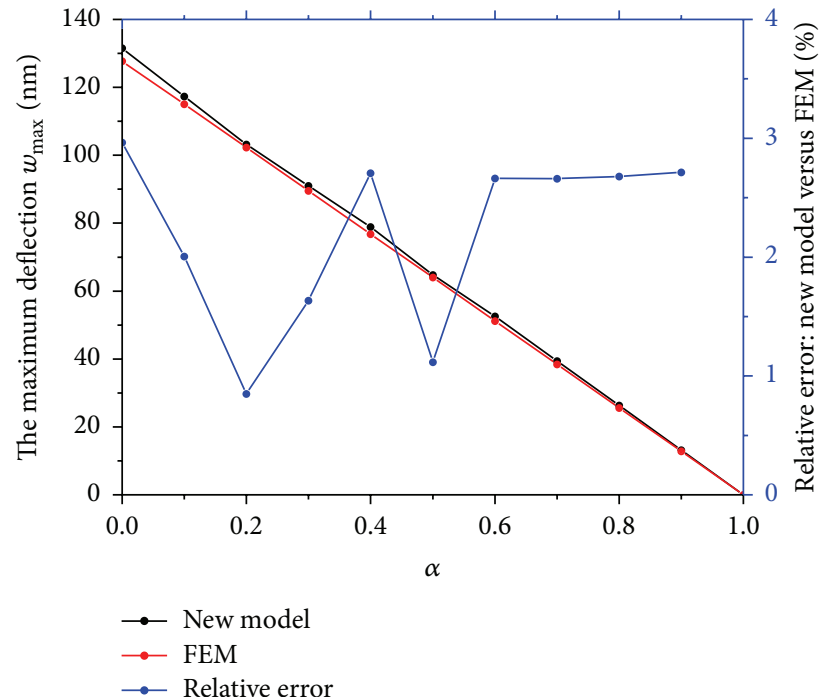

FIGURE 9: Maximum deflections and relative errors without biased voltage.

the pull-in ratio. Under different pressure ratios, the pull-in ratios and the relative errors are shown in Figure 11. Relevant data are listed in Table 4.

In Figure 11, the black line stands for the data given by our new model, the red line stands for FEM results, and the blue line represents the relative errors between the new model and FEM.

From Figure 11, it can be obtained that pull-in ratios decrease as the pressure ratios increase for both analytical and simulation results. When the pressure inside the sealed cavity is bigger ( $\alpha$ is bigger), the pull-in ratio is smaller, indicating that the moving part of the CMUT cell is harder to approach the pull-in point with a bigger pressure inside the sealed cavity. Also, the analytical results are in great agreement with FEM results, producing the relative errors ranging from $-6.986 \%$ to $1.612 \%$ (Table 4 ). As a matter of fact, FEMs like COMSOL are precise yet require more computing power and need more time to get the results, while the proposed new

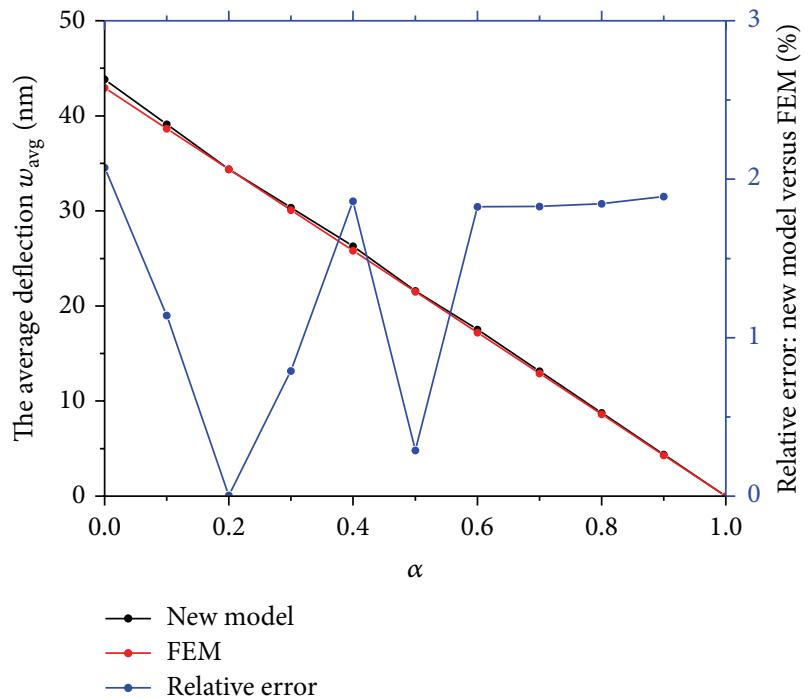

FIGURE 10: Average deflections and relative errors without biased voltage.

model is just several steps of calculations and more efficient to get the accurate results with acceptable relative errors. However, when compared to the relative errors produced in mechanical field only, the relative errors in electromechanical coupled fields are slightly bigger, indicating that the nonlinearity of the electrical force has brought bigger errors.

Notice that the tendency for analytical results is basically linear from $\alpha=0$ to $\alpha=1$, while the simulations show a smaller slope after $\alpha=0.5$. From $\alpha=0.5$ to $\alpha=1$, the pull-in ratios given by the new model decrease from 0.502 to 0.466 , yet the FEM results stay around 0.501 . This nonlinearity is maybe due to the electromechanical coupling effect aroused by the applied voltage. When the pressure inside the cavity is bigger than a certain value, the electromechanical coupling effect has bigger influence on the pull-in ratios, resulting in a series of similar pull-in ratios after that. This coupling effect also leads to bigger relative errors between the new model and FEM, which needs to be taken into consideration afterwards. 


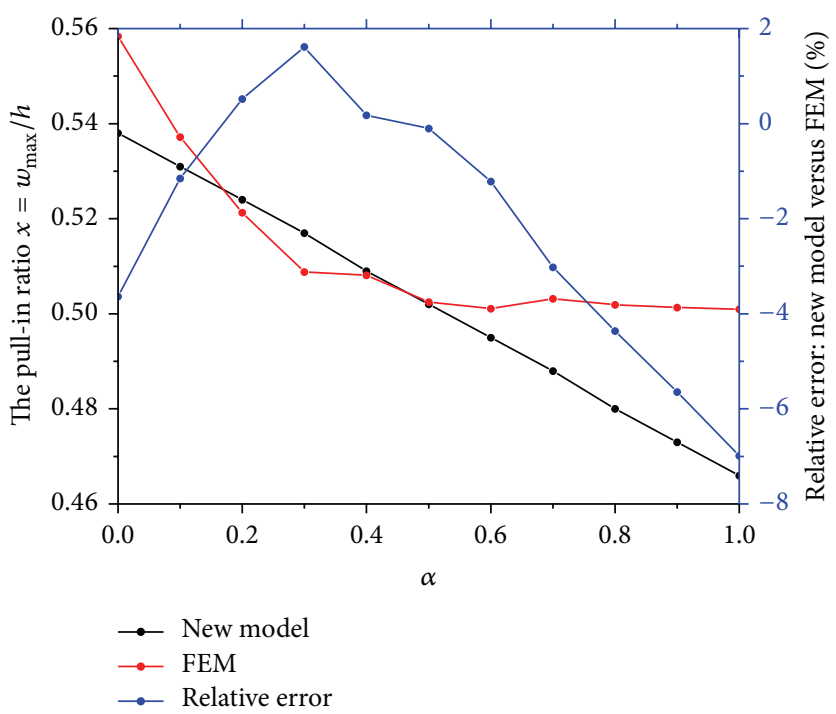

Figure 11: Pull-in ratios and relative errors under different pressure ratios.

TABLE 4: Pull-in voltage comparison of analytical to simulation results for the variety of pressure ratios.

\begin{tabular}{|c|c|c|c|c|c|c|}
\hline \multirow{2}{*}{$\alpha$} & \multicolumn{3}{|c|}{ The pull-in ratio $x=w_{\max } / h$} & \multicolumn{3}{|c|}{$V_{\text {pull-in }} / \mathrm{V}$} \\
\hline & New model & FEM & Relative error & New model & FEM & Relative error \\
\hline 0 & 0.538 & 0.558 & $-3.636 \%$ & 170.737 & 162.563 & $5.028 \%$ \\
\hline 0.1 & 0.531 & 0.537 & $-1.154 \%$ & 174.143 & 166.250 & $4.748 \%$ \\
\hline 0.2 & 0.524 & 0.521 & $0.518 \%$ & 177.559 & 170.273 & $4.279 \%$ \\
\hline 0.3 & 0.517 & 0.509 & $1.612 \%$ & 181.000 & 178.500 & $1.400 \%$ \\
\hline 0.4 & 0.509 & 0.508 & $0.177 \%$ & 184.461 & 188.135 & $-1.953 \%$ \\
\hline 0.5 & 0.502 & 0.503 & $-0.100 \%$ & 187.931 & 192.572 & $-2.410 \%$ \\
\hline 0.6 & 0.495 & 0.501 & $-1.217 \%$ & 191.412 & 195.426 & $-2.054 \%$ \\
\hline 0.7 & 0.488 & 0.503 & $-3.021 \%$ & 194.924 & 197.962 & $-1.535 \%$ \\
\hline 0.8 & 0.480 & 0.502 & $-4.363 \%$ & 198.447 & 200.523 & $-1.035 \%$ \\
\hline 0.9 & 0.473 & 0.501 & $-5.645 \%$ & 201.980 & 202.750 & $-0.380 \%$ \\
\hline 1 & 0.466 & 0.501 & $-6.986 \%$ & 205.529 & 204.558 & $0.475 \%$ \\
\hline
\end{tabular}

3.2.2. Pull-In Voltages Comparison. Commonly, when treated as rigid plates in open air, the CMUT cell has a uniform velocity profile across the surface. Combining the electrostatic force and the elastic force, the approximate pull-in voltage could be obtained. A case in point is the frequently cited literature [5]. Referring to [5], the pull-in voltage under such assumption is given by $V_{\text {pull-in }}=\sqrt{8 k h^{3} / 27 \varepsilon_{0} \pi R^{2}}$, without consideration of the pressure ratios. For the dimensions and material properties listed in Tables 1 and 2, the pullin voltage calculated by [5] is about $266.773 \mathrm{~V}$. The pull-in voltages obtained from our new model, the FEM, and [5] under different pressure ratios are all shown in Figure 12.

Relevant data are listed in Table 4.

In Figure 12, the black line stands for the data given by our new model, the red line stands for FEM results, the blue line represents the relative errors between the new model and FEM, and the pink line represents the pull-in voltage calculated by [5].
From Figure 12, it can be observed that pull-in voltages obtained from the new model and the FEM simulations both increase along with the pressure ratios, while the pull-in voltage calculated by [5] does not change with $\alpha$ since the pressures inside the sealed cavity are not taken into consideration. The analytical results predicted by the new model are in great agreement with FEM results, producing the relative errors ranging from $-2.410 \%$ to $5.028 \%$ (Table 4 ). However, the pullin voltage calculated by [5] is much larger than them. The analytical model used in [5] takes the CMUT as rigid plates in open air, resulting in the invariable and overestimated pullin voltage that could lead to improper characterization and experimental preparations. Again, notice that the tendency of the analytical results predicted by our new model is basically linear from $\alpha=0$ to $\alpha=1$, while the FEM simulations show fluctuations around the analytical results. Thus, the aforementioned electromechanical coupling effect does need to be taken into consideration for the accuracy of the present model. 


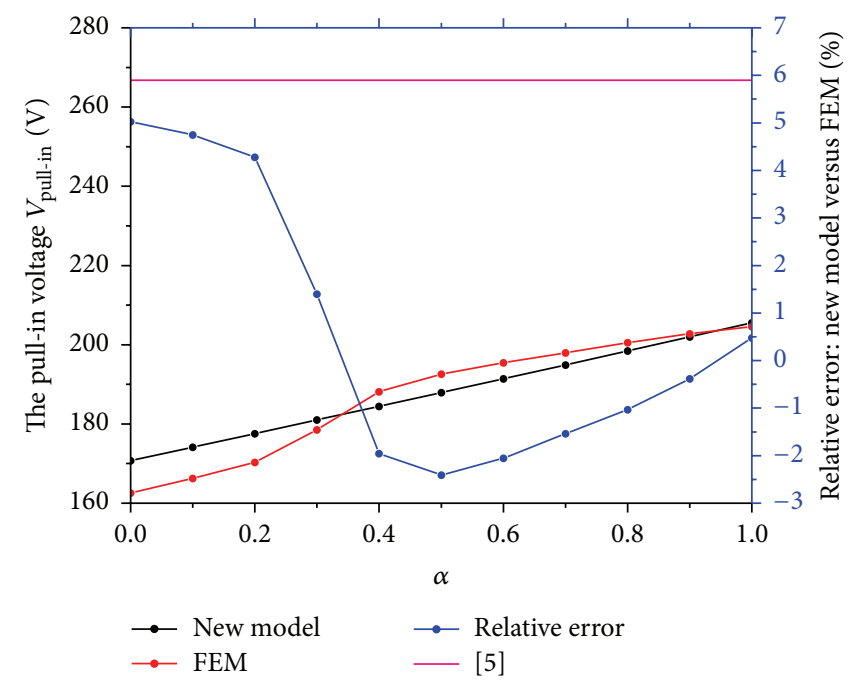

FIGURE 12: Pull-in voltages and relative errors under different pressure ratios.

\section{Conclusion}

This paper proposed a new analytical model for the pullin characteristics of the flat circular CMUT cell featuring sealed cavity based on the plate theory and Boyle's law. Not only did we point out that the existence of the pressure inside the sealed cavity cannot be omitted, but we also quantified the direct effect of the pressure ratios on the pullin phenomenon. The computational results of our analytical model were compared with the COMSOL simulations. The good agreement between them shows that our analytical model can approximate the CMUT's behavior fairly well. The work in this paper is summarized as follows.

(1) An improved analytical model for the pull-in characteristics of the flat circular CMUT cell featuring the sealed cavity has been presented. The strong relation between the pressures inside the sealed cavity and the pull-in characteristics is established for the first time. The derivation of this new model takes the top electrode into consideration and treats the CMUT as a flexible plate with fixed boundary conditions. The relations of the biased voltage $V$, the ratio $x$ of the central deflection to the effective gap distance, and the ratio $\alpha$ of the pressure inside the cavity to the ambient pressure are obtained.

(2) The validation and accuracy of the new model in mechanical field only have been obtained. The maximum and average deflections without biased voltage have been calculated by the new model and simulated by FEM. The maximum relative error is $2.964 \%$ for the maximum deflection and $2.073 \%$ for the average deflection, respectively. Thus, the mechanical analysis of the new model could accurately and efficiently predict the CMUT's vertical deflections.

(3) The validation and accuracy of the new model in coupled fields have been obtained. The pull-in characteristics under different pressure ratios have been verified by both the new model and FEM simulations. The maximum relative error is $6.986 \%$ for the pull-in ratios and $5.028 \%$ for the pullin voltages, respectively. In particular, the comparison of pull-in voltages among the present model, the FEM simulations, and the literature [5] that treats the CMUT as rigid plates leads to the conclusion that the fixed boundary conditions of the flexible thin plate are crucial to the model's validation and accuracy. Also the comparison does point out that the consideration of the pressures inside the sealed cavity is significant and necessary for the designing and modeling of the CMUT's pull-in analysis.

(4) Although our proposed process is quite precise during the analysis and validation, it has some limitations to be improved. The complex coupling nonlinearity causes bigger relative errors between the analytical pull-in characteristics and FEM results. In order to make the analytical model deliver smaller relative errors, the electromechanical coupling effect should be taken into consideration afterwards.

\section{Conflict of Interests}

The authors declare that there is no conflict of interests regarding the publication of this paper.

\section{Acknowledgment}

This work has been supported by the Young Scientists Fund of the National Natural Science Foundation of China (Grant no. 61201039).

\section{References}

[1] Ö. Oralkan, A. S. Ergun, J. A. Johnson et al., "Capacitive micromachined ultrasonic transducers: next-generation arrays for acoustic imaging?" IEEE Transactions on Ultrasonics, Ferroelectrics, and Frequency Control, vol. 49, no. 11, pp. 1596-1610, 2002.

[2] D. Gross, C. Coutier, M. Legros, A. Bouakaz, and D. Certon, "A cMUT probe for ultrasound-guided focused ultrasound targeted therapy," IEEE Transactions on Ultrasonics, Ferroelectrics, and Frequency Control, vol. 62, no. 6, pp. 1145-1160, 2015. 
[3] T. A. Emadi and D. A. Buchanan, "Design and characterization of a capacitive micromachined tranducer with a defectable bottom electrode," IEEE Electron Device Letters, vol. 36, no. 6, pp. 612-614, 2015.

[4] B. T. Khuri-Yakub and Ö. Oralkan, "Capacitive micromachined ultrasonic transducers for medical imaging and therapy," Journal of Micromechanics and Microengineering, vol. 21, no. 5, Article ID 054004, 2011.

[5] G. G. Yaralioglu, A. S. Ergun, B. Bayram, E. Hæggström, and B. T. Khuri-Yakub, "Calculation and measurement of electromechanical coupling coefficient of capacitive micromachined ultrasonic transducers," IEEE Transactions on Ultrasonics, Ferroelectrics, and Frequency Control, vol. 50, no. 4, pp. 449-456, 2003.

[6] B. Ahmad and R. Pratap, "Elasto-electrostatic analysis of circular microplates used in capacitive micromachined ultrasonic transducers," IEEE Sensors Journal, vol. 10, no. 11, pp. 1767-1773, 2010.

[7] E. Aydoğdu, A. Ozgurluk, A. Atalar, and H. Köymen, "Parametric nonlinear lumped element model for circular CMUTs in collapsed mode," IEEE Transactions on Ultrasonics, Ferroelectrics, and Frequency Control, vol. 61, no. 1, pp. 173-181, 2014.

[8] N. Apte, K. K. Park, and B. T. Khuri-Yakub, "Finite element analysis of CMUTs with pressurized cavities," in Proceedings of the IEEE International Ultrasonics Symposium (IUS '12), pp. 979-982, IEEE, Dresden, Germany, October 2012.

[9] B. Ahmad and R. Pratap, "Analytical evaluation of squeeze film forces in a CMUT with sealed air-filled cavity," IEEE Sensors Journal, vol. 11, no. 10, pp. 2426-2431, 2011.

[10] I. O. Wygant, M. Kupnik, and B. T. Khuri-Yakub, "Analytically calculating membrane displacement and the equivalent circuit model of a circular CMUT cell," in Proceedings of the IEEE International Ultrasonics Symposium (IUS '08), pp. 2111-2114, Beijing, China, November 2008.

[11] C. B. Doody, X. Y. Cheng, C. A. Rich, D. F. Lemmerhirt, and R. D. White, "Modeling and characterization of CMOS-fabricated capacitive micromachined ultrasound transducers," Journal of Microelectromechanical Systems, vol. 20, no. 1, pp. 104-118, 2011.

[12] M. L. Kuntzman, D. Kim, and N. A. Hall, "Microfabricated and experimental evaluation of a rotational capacitive micromachined ultrasonic transducers," Journal of Microelectromechanical Systems, vol. 24, no. 2, pp. 404-413, 2015.

[13] Y. Huang, A. Sanli Ergun, E. Hæggström, M. H. Badi, and B. T. Khuri-Yakub, "Fabricating capacitive micromachined ultrasonic transducers with wafer-bonding technology," Journal of Microelectromechanical Systems, vol. 12, no. 2, pp. 128-137, 2003.

[14] K. K. Park, H. Lee, M. Kupnik, and B. T. Khuri-Yakub, "Fabrication of capacitive micromachined ultrasonic transducers via local oxidation and direct wafer bonding," Journal of Microelectromechanical Systems, vol. 20, no. 1, pp. 95-103, 2011.

[15] W. Zhang, H. Zhang, Y. Wang, F. Du, S. Jin, and Z. Zeng, "Simulation characterization of CMUT with vented square membrane," in International Conference on Optical Instruments and Technology: Micro/Nano Photonics and Fabrication, vol. 9624 of Proceedings of SPIE, Beijing, China, May 2015.

[16] S. P. Timoshenko and S. Woinowsky-Krieger, Theory of Plates and Shells, McGraw-Hill, New York, NY, USA, 1959.

[17] D. Certon, F. Teston, and F. Patat, "A finite difference model for CMUT devices," IEEE Transactions on Ultrasonics, Ferroelectrics, and Frequency Control, vol. 52, no. 12, pp. 2199-2210, 2005.
[18] E. Ventsel and T. Krauthammer, Thin Plates and Shells, Marcel Deker, New York, NY, USA, 1st edition, 2001.

[19] Z. Wang, Microsystem Design and Fabrication, Tsinghua University Press, Beijing, China, 2008. 


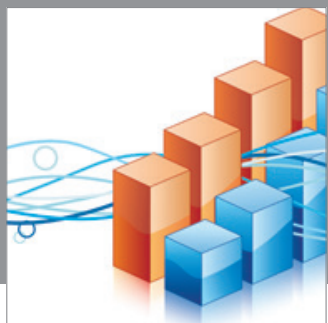

Advances in

Operations Research

mansans

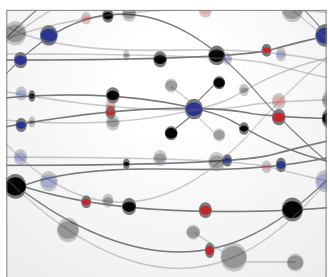

The Scientific World Journal
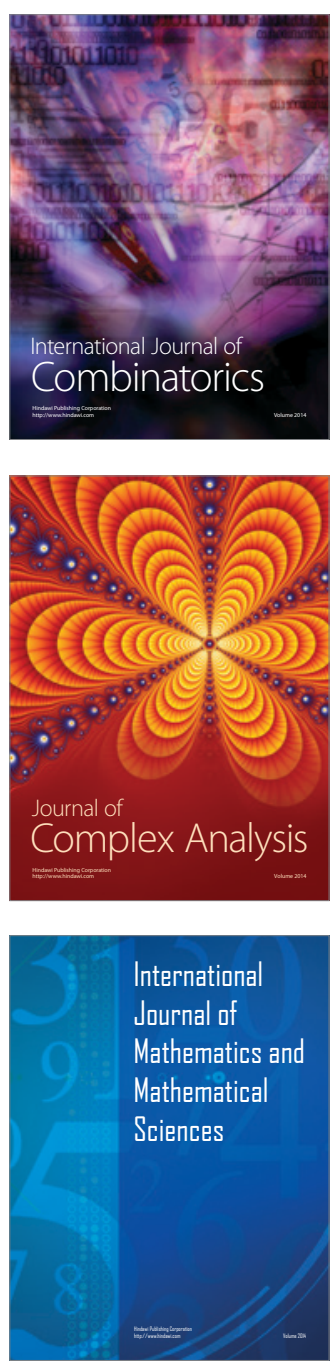
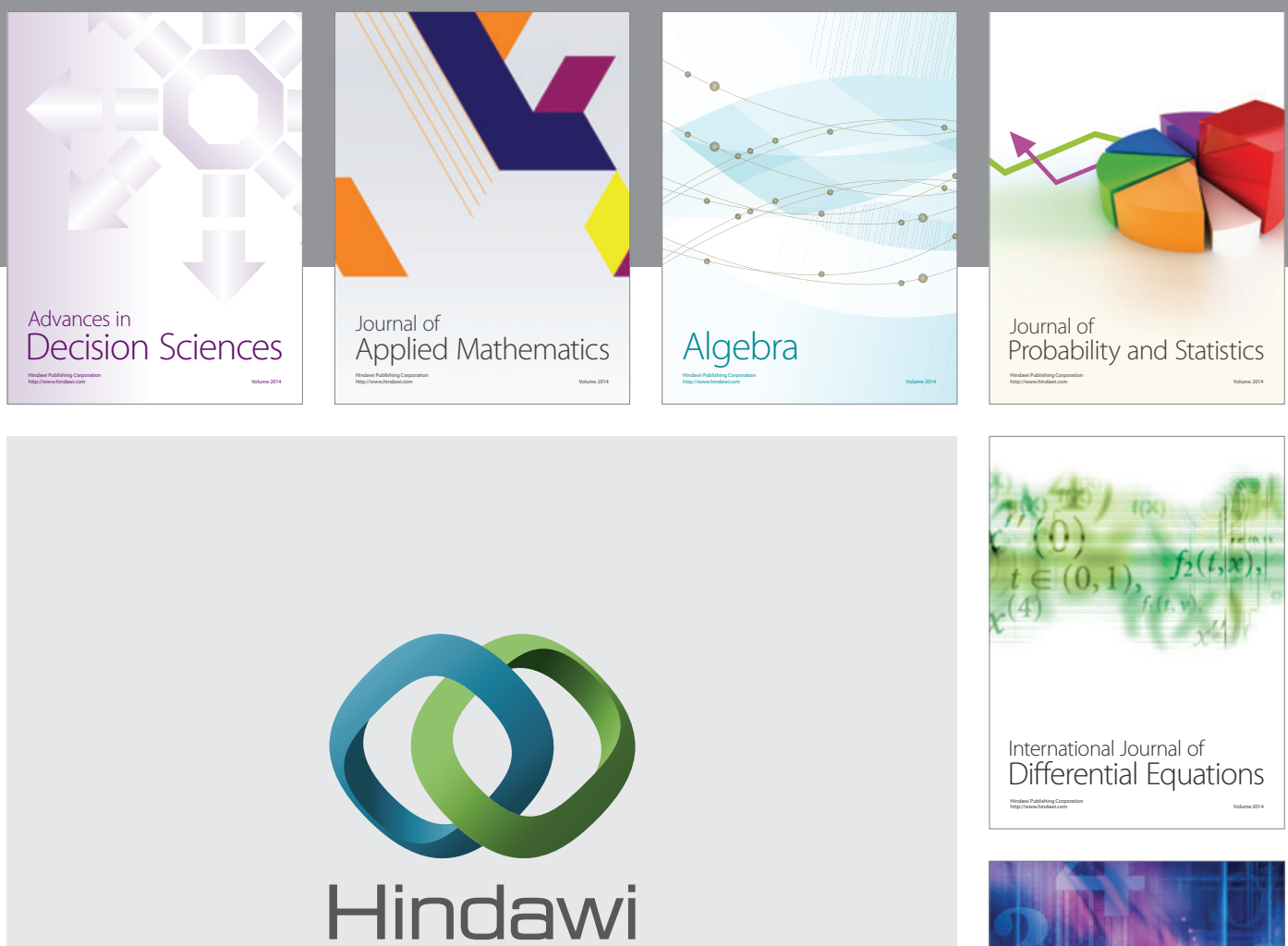

Submit your manuscripts at http://www.hindawi.com
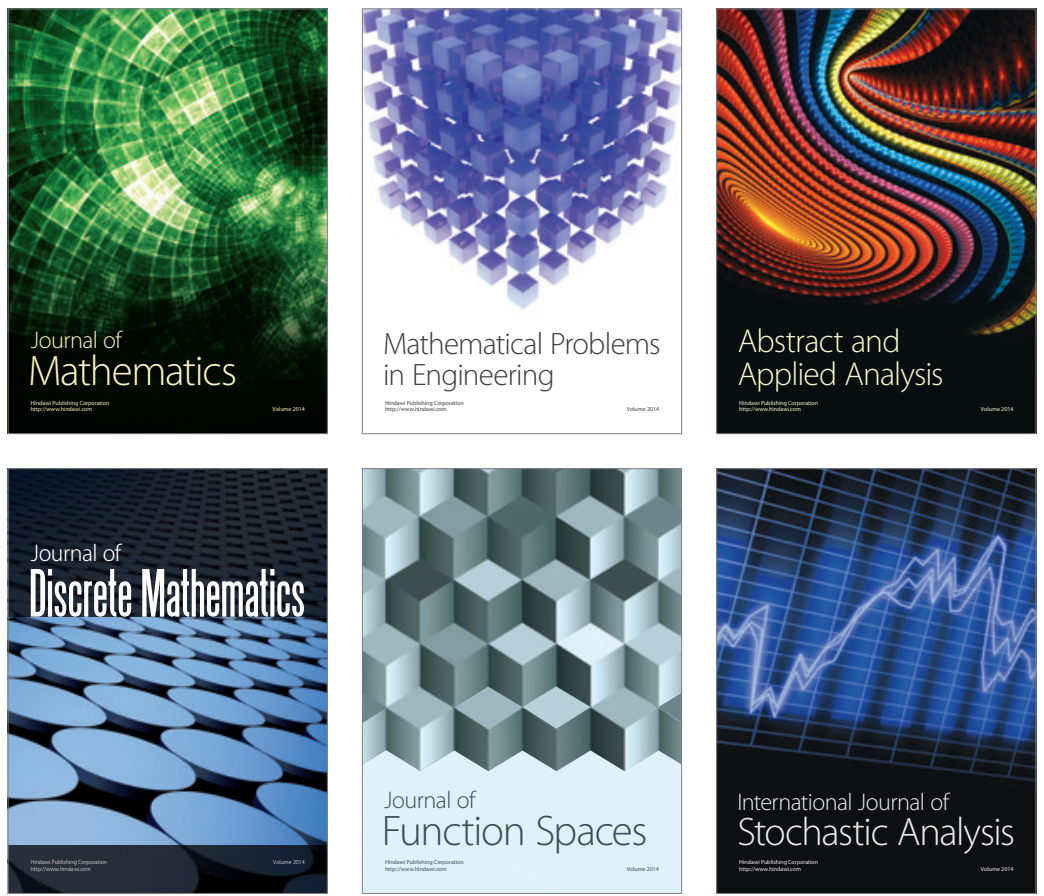

Journal of

Function Spaces

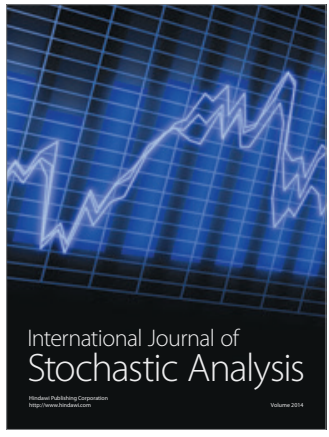

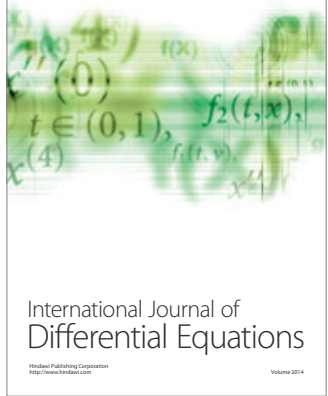
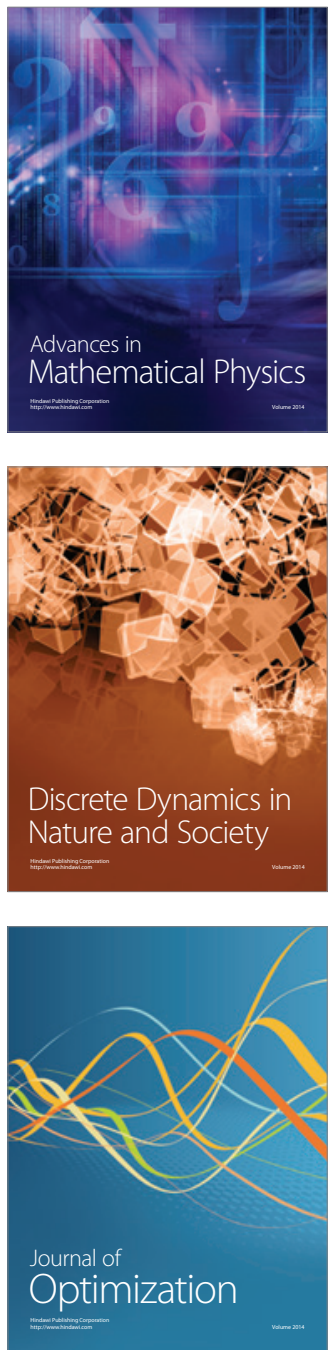